The Journal of

\title{
Mathematics and Computer Science
}

Available online at

\section{http://www.TIMCS.com}

The Journal of Mathematics and Computer Science Vol .5 No.4 (2012) 288-296

\section{Comparison Differential Transform Method with Homotopy Perturbation Method for Nonlinear Integral Equations}

\author{
Malihe Bagheri \\ Department of mathematic,Golestan Institute of Higher Education,Gorgan,Iran \\ Bagheri@golestan.ac.ir \\ Mahnaz Bagheri \\ Departmen of mathematic, Islamic Azad University, behshahr branch, Iran \\ m.bagheri@iaubs.ac.ir

\section{Ebrahim Miralikatouli} \\ Department of mathematic,Golestan Institute of Higher Education,Gorgan,Iran \\ emiralik@golestan.ac.ir
}

Received: February 2012, Revised: November 2012

Online Publication: December 2012

\begin{abstract}
In this study, an application of differential transform method (DTM) is applied to solve the second kind of nonlinear integral equations such that Volterra and Fredholm integral equations. If the equation considered has a solution in terms of the series expansion of known function, this powerful method catches the exact solution. Comparison is made between the homotopy perturbation and differential transform method. The results reveal that the differential transform method is very effective and simple.
\end{abstract}

Keywords: differential transform method, Integral equation, Volterra and Fredholm integral quations 


\section{INTRODUCTION}

The solutions of integral equations have a major role in the fields of science and engineering. When a plysical system is modeled under the differential sense; it finally gives a differential equation, an integral equation or integro-differentiol equation.

There are various techniques for solving integral equations, e.g. Adomian decomposition method (ADM) [1], Galerkin method [2], rationalized Haar functions method [3], He's homotopy perturbation method (HPM)[4] and variational iteration method(VIM)[5]. ADM is an analytical technique that evaluates the solution in the form of Adomian polynomials. This technique does not simplify or discretize the given problem and can be applied to both linear and non-linear problems. Galerkin and rationalized Haar functions methods are numerical techniques and there are numerous different approaches for the solution of integro-differential equations based on this method. HPM depends on the coupling of the classical perturbation method and the homotopy method in topology, firstly proposed by He [6]. VIM is an analytical method that can be applied to various types of linear and nonlinear problems. In this method, a correction functional is constructed by a general lagrange multiplier that can be identified optimally via the variational theory. Comparison among VIM, ADM and DTM for partial differential equations is presented by Bildik et al. [7]. Also,detailed analysis of various solution techniques is carried out in the review articles $[8,9]$.DTM is a semi analytical-numerical technique that depends on taylor series. It was first introduced by Zhou in a study about electrical circuits[10].It is possible to solve differential equations [11], difference equations[12], differentialdifference equation[13] and fractional equations[14] by using this method.

In this paper, an application of differential transform method (DTM) is applied to solve the second kind of nonlinear integral equations such that Volterra and Fredholm integral equations. The nonlinear Fredholm integral equations are given by

$\mathrm{u}(\mathrm{x})=\mathrm{f}(\mathrm{x})+\int_{0}^{1} \mathrm{~K}(\mathrm{x}, \mathrm{t})\{\mathrm{R}(\mathrm{u}(\mathrm{t}))+\mathrm{N}(\mathrm{u}(\mathrm{t}))\} \mathrm{dt}$.

and the nonlinear Volterra integral equations are given by

$$
\mathrm{u}(\mathrm{x})=\mathrm{f}(\mathrm{x})+\int_{0}^{\mathrm{x}} \mathrm{K}(\mathrm{x}, \mathrm{t})\{\mathrm{R}(\mathrm{u}(\mathrm{t}))+\mathrm{N}(\mathrm{u}(\mathrm{t}))\} \mathrm{dt} .
$$


$\mathrm{u}(\mathrm{t})$ is a unknown function that will be determined $\mathrm{K}(\mathrm{x}, \mathrm{t})$ is the kernel of the integral equation, $\mathrm{f}(\mathrm{x})$ is an analytic function, $\mathrm{R}(\mathrm{u})$ and $\mathrm{N}(\mathrm{u})$ are linear and nonlinear functions of $\mathrm{u}$, respectively[15].

In this letter, we apply differential transform method (DTM) to solve the second kind of nonlinear integral equations such that Volterra and Fredholm integral equations. Then compare this method with the modification of homotopy perturbation method (HPM). The results reveal that the differential transform method is very effective and simple.

\section{DIFFERENTIAL TRANSFORM METHOD}

The transformation of the kth derivative of a function in one variable is as follows:

$\mathrm{F}(\mathrm{k})=\frac{1}{\mathrm{k} !}\left[\frac{\mathrm{d}^{\mathrm{k}} \mathrm{f}(\mathrm{x})}{\mathrm{dx}^{\mathrm{k}}}\right]_{\mathrm{x}=\mathrm{x}_{0}}$

Where $f(x)$ is the original function and $F(k)$ is the transformed function. The differential inverse transformation of $\mathrm{F}(\mathrm{k})$ is defined as

$f(x)=\sum_{k=0}^{\infty} F(k)\left(x-x_{0}\right)^{k}$.

From Eqs.(1) and (2), we get

$f(x)=\sum_{k=0}^{\infty} \frac{1}{k !}\left[\frac{d^{\mathrm{k}} \mathrm{f}(\mathrm{x})}{d \mathrm{x}^{\mathrm{k}}}\right]_{\mathrm{x}=\mathrm{x}_{0}}\left(\mathrm{x}-\mathrm{x}_{0}\right)^{\mathrm{k}}$.

Which implies the concept of differential transform is derived from Taylor series expansion. In real applications, the function $\mathrm{f}(\mathrm{x})$ is expressed by a finite series and Eq (2) can be written as

$f(x)=\sum_{k=0}^{N} F(k)\left(x-x_{0}\right)^{k}$.

Here $\mathrm{N}$ is decided by the convergence of natural frequency.

The following theorems that can be deduced form Eqs. (1) and (2) are given as:

Theorem 2. 1. If $f(x)=g(x) \pm h(x)$, then $F(k)=G(k) \pm H(k)$.

Theorem2. 2. $\operatorname{Iff}(\mathrm{x})=\mathrm{cg}(\mathrm{x})$, then $\mathrm{F}(\mathrm{k})=\mathrm{cG}(\mathrm{k})$, where $\mathrm{c}$ is a constant.

Theorem2. 3. If $f(x)=\frac{d^{n} g(x)}{d x^{n}}$, then $F(k)=\frac{(k+n) !}{k !} G(k+n)$.

Theorem2. 4. If $\mathrm{f}(\mathrm{x})=\mathrm{g}(\mathrm{x}) \mathrm{h}(\mathrm{x})$, then $\mathrm{F}(\mathrm{k})=\sum_{\mathrm{k}_{1}=0}^{\mathrm{k}} \mathrm{G}\left(\mathrm{k}_{1}\right) \mathrm{H}\left(\mathrm{k}-\mathrm{k}_{1}\right)$.

Theorem2. 5. If $(\mathrm{x})=\mathrm{x}^{\mathrm{n}}$, then $\mathrm{F}(\mathrm{x})=\delta(\mathrm{k}-\mathrm{n})$,

where $\delta(k-n)=\left\{\begin{array}{cc}1 & k=n \\ 0 & k \neq n\end{array}\right.$. 
Theorem2. 6. If $f(x)=g_{1}(x) g_{2}(x) \ldots g_{n-1}(x) g_{n}(x)$

then $\mathrm{F}(\mathrm{k})=\sum_{\mathrm{k}_{\mathrm{n}-1}=0}^{\mathrm{k}} \sum_{\mathrm{k}_{\mathrm{n}-2}=0}^{\mathrm{k}_{\mathrm{n}} \ldots} \sum_{\mathrm{k}_{2}=0}^{\mathrm{k}_{3}} \sum_{\mathrm{k}_{1}=0}^{\mathrm{k}_{2}} \mathrm{G}_{1}\left(\mathrm{k}_{1}\right) \mathrm{G}_{2}\left(\mathrm{k}_{2}-\mathrm{k}_{1}\right) \ldots \mathrm{G}_{\mathrm{n}-1}\left(\mathrm{k}_{\mathrm{n}-1}-\right.$ $\left.\mathrm{k}_{\mathrm{n}-2}\right) \mathrm{G}_{\mathrm{n}}\left(\mathrm{k}-\mathrm{k}_{\mathrm{n}-1}\right)$

Theorem2. 7. If $f(x)=\int_{x_{0}}^{x} g(t) d t$, then $F(k)=\frac{G(k-1)}{k}$, where $k \geq 1$.

Theorem2. 8. If $\mathrm{f}(\mathrm{x})=\int_{\mathrm{x}_{0}}^{\mathrm{x}} \int_{\mathrm{x}_{0}}^{\mathrm{x}_{\mathrm{n}}-1} \ldots \int_{\mathrm{x}_{0}}^{\mathrm{x}_{2}} \int_{\mathrm{x}_{0}}^{\mathrm{x}_{1}} \mathrm{~g}(\mathrm{t}) \mathrm{dtdx_{1 }} d \mathrm{dx}_{2} \ldots d \mathrm{x}_{\mathrm{n}-1}$ then $\mathrm{F}(\mathrm{k})=\frac{(\mathrm{k}-\mathrm{n}) !}{\mathrm{k} !} \mathrm{G}(\mathrm{k}-\mathrm{n})$, where $\mathrm{k} \geq \mathrm{n}$.

Theorem2. 9. If $\mathrm{f}(\mathrm{x})=\mathrm{g}(\mathrm{x}) \int_{\mathrm{x}_{0}}^{\mathrm{x}} \mathrm{h}(\mathrm{t}) \mathrm{dt}$, then $\mathrm{F}(\mathrm{k})=\frac{1}{\mathrm{k}} \sum_{\mathrm{k}_{1}=1}^{\mathrm{k}} \frac{1}{\mathrm{k}_{1}} \mathrm{G}\left(\mathrm{k}-\mathrm{k}_{1}\right) \mathrm{H}\left(\mathrm{k}_{1}-\right.$ $1)$, where $\mathrm{k} \geq 1$.

Theorem2. 10. If $\mathrm{f}(\mathrm{x})=\int_{\mathrm{x}_{0}}^{\mathrm{x}} \mathrm{g}_{1}(\mathrm{t}) \mathrm{g}_{2}(\mathrm{t}) \mathrm{dt}$, then $\mathrm{F}(\mathrm{x})=\frac{1}{\mathrm{k}} \sum_{\mathrm{k}_{1}=0}^{\mathrm{k}-1} \mathrm{G}_{1}\left(\mathrm{k}_{1}\right) \mathrm{G}_{2}(\mathrm{k}-$ $\mathrm{k}_{1}-1$ ), where $\mathrm{k} \geq 1$.

Theorem2.11. If $\mathrm{f}(\mathrm{x})=\int_{\mathrm{x}_{0}}^{\mathrm{x}} \mathrm{g}_{1}(\mathrm{t}) \mathrm{g}_{2}(\mathrm{t}) \ldots \mathrm{g}_{\mathrm{n}-1}(\mathrm{t}) \mathrm{dt}$ then

$$
\begin{aligned}
\mathrm{F}(\mathrm{k})=\frac{1}{\mathrm{k}} \sum_{\mathrm{k}_{\mathrm{n}-1}=0}^{\mathrm{k}-1} \sum_{\mathrm{k}_{\mathrm{n}-2}=0}^{\mathrm{k}_{\mathrm{n}}} \ldots \sum_{\mathrm{k}_{2}=0}^{\mathrm{k}_{3}} \sum_{\mathrm{k}_{1}=0}^{\mathrm{k}_{2}} \mathrm{G}_{1}\left(\mathrm{k}_{1}\right) \\
\mathrm{G}_{2}\left(\mathrm{k}_{2}-\mathrm{k}_{1}\right) \ldots \mathrm{G}_{\mathrm{n}-1}\left(\mathrm{k}_{\mathrm{n}-1}-\mathrm{k}_{\mathrm{n}-2}\right) \mathrm{G}_{\mathrm{n}}\left(\mathrm{k}-\mathrm{k}_{\mathrm{n}-1}-1\right)
\end{aligned}
$$

Theorem2. 12. If

$f(x)=\left[g_{1}(x) g_{2}(x) \ldots g_{n-1}(x) g_{n}(x)\right] \times \int_{x_{0}}^{x} h_{1}(x) h_{2}(x) \ldots h_{m-1}(x) h_{m}(x) d t$ then

$$
\mathrm{F}(\mathrm{k})=\sum_{\substack{\left.\mathrm{m}+\mathrm{n}-1 \\=1 \\ \mathrm{k}_{\mathrm{m}+\mathrm{n}-2}=1 \\-\mathrm{k}_{\mathrm{m}-2}\right) \mathrm{H}_{\mathrm{m}}\left(\mathrm{k}_{\mathrm{m}}-\mathrm{k}_{\mathrm{m}-1}\right)}}^{\mathrm{k}} \sum_{\mathrm{k}_{2}=1}^{\mathrm{k}_{\mathrm{m}+\mathrm{n}-1}} \sum_{\mathrm{k}_{1}}^{\mathrm{k}_{3}} \frac{1}{\mathrm{k}_{\mathrm{m}}} \mathrm{H}_{1}\left(\mathrm{k}_{1}-1\right) \mathrm{H}_{2}\left(\mathrm{k}_{2}-\mathrm{k}_{1}\right) \ldots \mathrm{H}_{\mathrm{m}-1}\left(\mathrm{k}_{\mathrm{m}-1}\right.
$$

$\mathrm{G}_{1}\left(\mathrm{k}_{\mathrm{m}+1}-\mathrm{k}_{\mathrm{m}}\right) \mathrm{G}_{2}\left(\mathrm{k}_{\mathrm{m}+2}-\mathrm{k}_{\mathrm{m}+1}\right) \ldots \mathrm{G}_{\mathrm{n}-1}\left(\mathrm{k}_{\mathrm{m}+\mathrm{n}-1}-\mathrm{k}_{\mathrm{m}+\mathrm{n}-2}\right) \mathrm{G}_{\mathrm{n}}\left(\mathrm{k}-\mathrm{k}_{\mathrm{m}+\mathrm{n}-1}\right)$.

The evolution of a definite integral can be obtained from theorem 11 and Eq.(2) in the most general form as follows:

$\int_{a}^{b} g_{1}(t) g_{2}(t) \ldots g_{n-1}(t) d t=$ $\sum_{\mathrm{k}=1}^{\infty}\left\{\frac{1}{\mathrm{k}}\left[\left(\mathrm{b}-\mathrm{x}_{0}\right)^{\mathrm{k}}-\left(\mathrm{a}-\mathrm{x}_{0}\right)^{\mathrm{k}}\right]\left[\sum_{\mathrm{k}_{\mathrm{n}-1}=0}^{\mathrm{k}-1} \sum_{\mathrm{k}_{\mathrm{n}-2}=0}^{\mathrm{k}_{\mathrm{n}-1}} \cdots \sum_{\mathrm{k}_{2}=0}^{\mathrm{k}_{3}} \sum_{\mathrm{k}_{1}=0}^{\mathrm{k}_{2}} \mathrm{G}_{1}\left(\mathrm{k}_{1}\right) \mathrm{G}_{2}(\mathrm{k}-\right.\right.$ $\left.\mathrm{k}_{1}\right) \ldots$

$$
\left.\left.\mathrm{G}_{\mathrm{n}-1}\left(\mathrm{k}_{\mathrm{n}-1}-\mathrm{k}_{\mathrm{n}-2}\right) \mathrm{G}_{\mathrm{n}}\left(\mathrm{k}-\mathrm{k}_{\mathrm{n}-1}-1\right)\right]\right\}
$$

The proof of Theorems (2.1) to (2.12) is available in (Odibat,Z.M.,2008)[16]. 


\section{APPLICATION AND NUMERICAL RESULTS}

This section contained three examples of non-linear Volterra and Fredholm integral equations of the second kind.

Example 3.1. Consider non-linear Fredholm integral equation in the following form:

$\mathrm{u}(\mathrm{x})=\sinh (\mathrm{x})-1+\int_{0}^{1}\left(\cosh (\mathrm{t})^{2}-\mathrm{u}^{2}(\mathrm{t})\right) \mathrm{dt}$

Eq.(5) is transformed by using Theorem (2.12) as follows:

$\mathrm{U}(\mathrm{k})=\mathrm{G}(\mathrm{k})-\delta(\mathrm{k})+\alpha_{1} \delta(\mathrm{k})-\alpha_{2} \delta(\mathrm{k})$

Where

$$
\alpha_{1}=\int_{0}^{1} \cosh (t)^{2} d t, \alpha_{2}=\int_{0}^{1} u(t)^{2} d t
$$

$\mathrm{F}(\mathrm{k})$ and $\mathrm{G}(\mathrm{k})$ are differential transform method of $\sinh (\mathrm{x})$ and $\cosh (\mathrm{x})$ respectively. These can be deduced by Eq.(1) as follows:

$$
\begin{aligned}
& \mathrm{F}(\mathrm{k})= \begin{cases}\frac{1}{\mathrm{k} !} & \text { if } \mathrm{k}=\text { odd } \\
0 & \text { if } \mathrm{k}=\text { even }\end{cases} \\
& \text { and } G(k)= \begin{cases}0 & \text { if } \mathrm{k}=\text { odd } \\
\frac{1}{\mathrm{k} !} & \text { if } \mathrm{k}=\text { even }\end{cases}
\end{aligned}
$$

Utilizing Eq. (5), (6) and (7), one can show that the following equalities hold for $\alpha_{1}$ and $\alpha_{2}$.

$$
\begin{aligned}
& \alpha_{1}=\sum_{\mathrm{k}=1}^{\infty}\left\{\frac{1}{\mathrm{k}} \sum_{\mathrm{k}_{1}=0}^{\mathrm{k}-1}\left(\mathrm{~F}\left(\mathrm{k}_{1}\right) \mathrm{F}\left(\mathrm{k}-\mathrm{k}_{1}-1\right)\right)\right\} \\
& \alpha_{2}=\sum_{\mathrm{k}=1}^{\infty}\left\{\frac{1}{\mathrm{k}} \sum_{\mathrm{k}_{1}=0}^{\mathrm{k}-1}\left(\mathrm{U}\left(\mathrm{k}_{1}\right) \mathrm{U}\left(\mathrm{k}-\mathrm{k}_{1}-1\right)\right)\right\}
\end{aligned}
$$

By using the inverse transformation rule in Eq.(4) the following eries solution are evaluated

$\mathrm{u}(\mathrm{x})=\left(-1+\alpha_{1}-\alpha_{2}\right)+\frac{1}{1 !} \mathrm{x}+\frac{1}{3 !} \mathrm{x}^{3}+\frac{1}{5 !} \mathrm{x}^{5}+\cdots$

Solving Eq.(10) and (11) by taking $U(\mathrm{k})$, we obtained the following result $\alpha_{1}=1, \alpha_{2}=0$.

Substitute $\alpha_{1}$ and $\alpha_{2}$ into (12) to obtain the exact solution as $\mathrm{u}(\mathrm{x})=\sinh (\mathrm{x})$

Example 3.2. Consider the non-linear Fredholm integral equation in the following form:

$\mathrm{u}(\mathrm{x})=\cos \mathrm{x}-\mathrm{x}+\int_{0}^{1} \mathrm{x}\left(\mathrm{u}(\mathrm{t})^{2}-\sin (\mathrm{t})^{2}\right) d \mathrm{t}$

Appling differential transform on Eq.(13) and using theorem (2.12) ,we obtained

$\mathrm{U}(\mathrm{k})=\frac{1}{\mathrm{k} !} \cos \left(\frac{\mathrm{k} \pi}{2}\right)-\delta(\mathrm{k}-1)+\delta(\mathrm{k}-1)\left[\alpha_{1}-\alpha_{2}\right]$

Where $\alpha_{1}=\int_{0}^{1} \mathrm{u}(\mathrm{t})^{2} \mathrm{dt}, \alpha_{2}=\int_{0}^{1} \sin (\mathrm{t})^{2} \mathrm{dt}$ 
Utilizing Eq.(15) one can show that the following equalities hold for $\alpha_{1}$ and $\alpha_{2}$.

$\alpha_{1}=\sum_{\mathrm{k}=1}^{\infty}\left\{\frac{1}{\mathrm{k}} \sum_{\mathrm{k}_{1}=0}^{\mathrm{k}-1}\left(\mathrm{U}\left(\mathrm{k}_{1}\right) \mathrm{U}\left(\mathrm{k}-\mathrm{k}_{1}-1\right)\right)\right\}$

$\alpha_{2}=\sum_{\mathrm{k}=1}^{\infty}\left\{\frac{1}{\mathrm{k}} \sum_{\mathrm{k}_{1}=0}^{\mathrm{k}-1}\left(\mathrm{~F}\left(\mathrm{k}_{1}\right) \mathrm{F}\left(\mathrm{k}-\mathrm{k}_{1}-1\right)\right)\right\}$

Where $\mathrm{F}(\mathrm{k})=\frac{1}{\mathrm{k} !}$

By using the inverse transformation rule in Eq. (4) the following series solution are evaluated

$\mathrm{u}(\mathrm{x})=1+\left(-1+\alpha_{1}-\alpha_{2}\right) \mathrm{x}+\frac{1}{2 !} \mathrm{x}^{2}+\frac{1}{4 !} \mathrm{x}^{4}-\frac{1}{6 !} \mathrm{x}^{6}$

Solving Eqs(16) and (17) by taking $U(\mathrm{k})$, we obtained the following result $\alpha_{1}=1 \quad, \alpha_{2}=0$

Substitute $\alpha_{1}$ and $\alpha_{2}$ into (18) to obtain the exact solution as $\mathrm{u}(\mathrm{x})=\cos (\mathrm{x})$

Example 3.3. Consider the non-linear Fredholm integral equation in the following form:

$\mathrm{u}(\mathrm{x})=\mathrm{e}^{\mathrm{x}}-\frac{1}{2} \mathrm{x}\left(\mathrm{e}^{2 \mathrm{x}}-1\right)-\int_{0}^{\mathrm{x}} \mathrm{xu}(\mathrm{t})^{2} \mathrm{dt}$

Appling the differential transform on Eq.(3.15), we obtained

$$
\begin{aligned}
\mathrm{U}(\mathrm{k})=\frac{1}{\mathrm{k} !}+\sum_{\mathrm{k}_{1}}^{\mathrm{k}} \delta\left(\mathrm{k}_{1}-1\right) \frac{2^{\mathrm{k}-\mathrm{k}_{1}}}{\left(\mathrm{k}-\mathrm{k}_{1}\right) !}-\frac{1}{2} \delta(\mathrm{k}-1) & \\
& -\sum_{\mathrm{k}_{2}=1}^{\mathrm{k}} \sum_{\mathrm{k}_{1}=1}^{\mathrm{k}_{2}} \mathrm{U}\left(\mathrm{k}_{1}-1\right) \mathrm{U}\left(\mathrm{k}-\mathrm{k}_{1}\right) \delta(\mathrm{k}-1)
\end{aligned}
$$

Note that the transformation of integrals are considered for $\mathrm{k} \geq 1$.we have $\mathrm{U}(0)=1, \mathrm{U}(1)=1, \mathrm{U}(2)=\frac{1}{2 !}, \mathrm{U}(3)=\frac{1}{3 !}, \ldots$

And so on, in general $\mathrm{U}(\mathrm{k})=\frac{1}{\mathrm{k} !}$. Subsititute all $\mathrm{U}(\mathrm{k})$ into Eq. (4) to obtained the exact solution as $\mathrm{u}(\mathrm{x})=\mathrm{e}^{\mathrm{x}}$

\section{FIGURES}

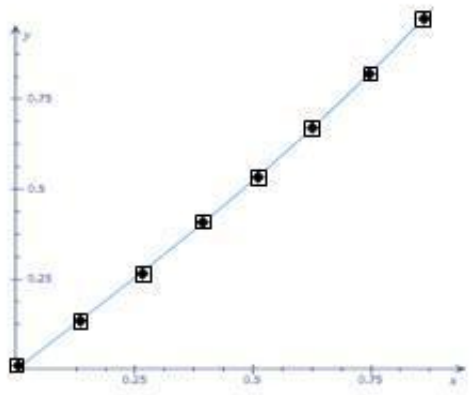

Fig .1.the numerical result of the exact solution $u(x)$ of Eq.(5) with DTM wich

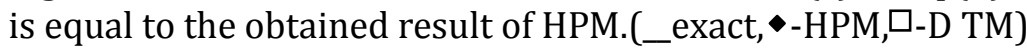




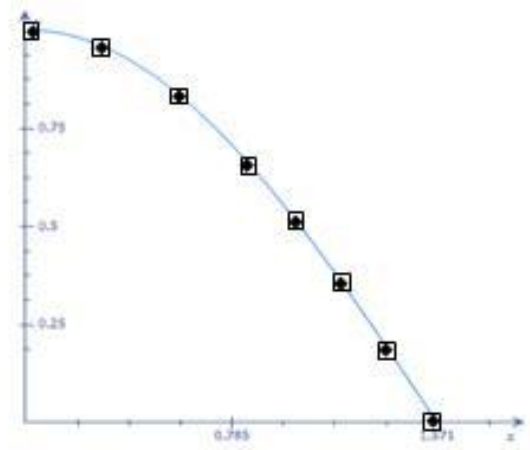

Fig .2.the numerical result of the exact solution $\mathrm{u}(\mathrm{x})$ of Eq. (13) with DTM wich is equal to the obtained result of HPM. (_exact, -HPM $\square$-DTM).

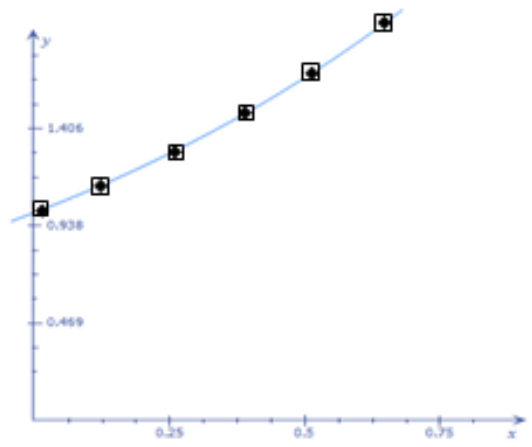

Fig.3.the numerical result of the exact solution $u(x)$ of Eq.(19) with DTM wich is equal to the obtained result of HPM.(__exact, •-HPM, $\square-D T M)$

\section{CONCLUSION}

In this paper, Differential transform method has been used for finding the solutions of nonlinear of Volterra and Feredholm integral equations .comparing the DTM with the exact solution by HPM that they have got the same answer. These results are plotted in Figures 1 to 3, as well as the exact solutions. It can be concluded that, DTM is a very powerful and efficient technique, for finding exact solutions for nonlinear problems in comparison with other methods. 


\section{REFERENCES}

[1] J. Biazar, E. Babolian, R. Islam, Solution of a system of Volterra integral equations of the first kind by Adomian method, Appl. Math. Comput. 139 (2003) 249-258.

[2]K. Maleknejad, M. Tavassoli Kajani, solving linear integro-differential equation system by Galerkin methods with hybrid functions, Appl. Math. Comput. 159 (2004) 603-612.

[3] K. Maleknejad, F. Mirzaee, S. Abbasbandy, solving linear integrodifferential equations system by using rationalized Haar functions method, Appl. Math. Comput. 155 (2004) 317-328.

[4] J. Biazar, H. Ghazvini. M. Eslami, He's homotopy perturbation method for system of integral equations Chaos solitons. Fractal,(2007).06.001.

[5] S. Q. Wang, J. H. He,Variational iteration method for solving integrodifferential equations, Phys. Lett.A. 367 (2007) 188-191.

[6] J.H. He Homotopy technique and a perturbation technique for non-linear problems, Int J. Non linear Mech. 35 (2000)37-43.

[7] N. Bildik, A. Konuralp, Two-dimensional differential transform method, Adomian's decomposition method, and variational iteration method for partial differential equations, Int.J. Comput. Math. 83 (2006)973-987.

[8] J.H. He, New interpretation of homotopy perturbation method,Internat. J. Modern Phys. B. 20 (2006) 2561-2568.

[9] J.H. He, Some asymptotic methods for strongly nonlinear equations, Internat. J. Modern Phys. B. 20 (2006) 1141-1199.

[10] J.K. Zhou, Differential Transform and its Application for Electrical Circuits,Huazhong University Press, Wuhan, China, 1968.

[11] 0. Ozdemir, M.o. Kaya, Flapwise bending vibration analysis of a rotating tapered cantilever Bernoulli-Euler bean by differential transform method, J. Sound Vid. 289 (2006) 413-420.

[12] A. Arikoglu,I.Ozkol, Solution of difference equations by using differential transform method, Appl. Math. Comput. 174 (2006) 1216-1228.

[13] A. Arikoglu, I. Ozkol, Solution of differential-difference equations by using differential transform method, Appl. Math. Comput. 181 (2006) 153162.

[14] A. Arikoglu, I. Ozkol, Solution of fractial differential equations by using differential transform method, Chaos Solitone. Fract. 34 (2007)1473-1481. 
[15] D. D. Ganji, G. A. Afrouzi, H. Hosseinzadeh, R. A. Talarposhti, Application of homotopy-perturbation method to the second kind of nonlinear integral equations, Phys. Lett.A. 371 (2007) 20-25.

[16] Z.Odibat, Differential Transform method for solving Volterra integral equations with separable kernels, Mathematics computational modeling, 48(2008) 1144-1149 